\title{
DEFICIENCIES OF AN ENTIRE ALGEBROID FUNCTION OF FINITE ORDER
}

\author{
By TSuneo SAto
}

§1. Recently Niino-Ozawa [1], [2] has established some curious results for a two- or three- or four-valued entire algebroid function. A typical theorem is the following:

THEOREM. Let $f(z)$ be a two-valued entire transcendental algebroid function and $a_{1}, a_{2}$ and $a_{3}$ be different finite numbers satisfying

$$
\delta\left(a_{1}, f\right)+\delta\left(a_{2}, f\right)+\delta\left(a_{3}, f\right)>2 .
$$

Then at least one of $\left\{a_{j}\right\}$ is a Picard exceptional value of $f$.

This result discloses the remarkable fact that the condition only on the deficiencies implies the existence of a Picard exceptional value in the two-valued case and there is a big gap between the distribution of deficiencies of entire algebroid functions and that of one-valued entire functions. follows:

In this paper we shall relax somewhat the condition on the deficiencies as

THEOREM 1. Let $f(z)$ be a two-valued entire transcendental algebroid function of finite order by an irreducible equation

$$
F(z, f) \equiv f^{2}+A_{1} f+A_{0}=0,
$$

where $A_{1}$ and $A_{0}$ are entire functions in $|z|<\infty$. Let $a_{1}, a_{2}$ and $a_{3}$ be three different finite numbers satisfying

$$
\Delta\left(a_{1}, f\right)+\delta\left(a_{2}, f\right)+\delta\left(a_{3}, f\right)>2,
$$

where $\delta(a, f)$ and $\Delta(a, f)$ indicate the Nevanlinna-Selberg deficiency and Valiron deficiency of $f$ at a respectively. Then at least one of $\left\{a_{j}\right\}$ is a Picard exceptional value of $f$ or more precisely it occurs either

$$
\begin{array}{ll}
\delta\left(a_{1}, f\right)=1, & \delta\left(a_{2}, f\right)=\delta\left(a_{3}, f\right)>\frac{1}{2} \quad \text { or } \\
\delta\left(a_{2}, f\right)=1, \quad \Delta\left(a_{1}, f\right)=\Delta\left(a_{3}, f\right)>\frac{1}{2} .
\end{array}
$$

Received January 14, 1971. 
Further if there is another deficiency of $f$ at $a_{4}$ then

$(\text { a })^{\prime} \quad \delta\left(a_{4}, f\right) \leqq 1-\delta\left(a_{2}, f\right)$ or

(b) $\delta\left(a_{4}, f\right) \leqq 1-\Delta\left(a_{1}, f\right)$ corresponding to the cases (a) or (b).

THEOREM 2. Let $f(z)$ be a three-valued transcendental entire algebroid function of finite order defined by an irreducible equation

$$
F(z, f) \equiv f^{3}+A_{2} f^{2}+A_{1} f+A_{0}=0,
$$

where $A_{2}, A_{1}$ and $A_{0}$ are entire functions. Let $a_{1}, a_{2}, a_{3}$ and $a_{4}$ be four different finite numbers satisfying

$$
\Delta\left(a_{1}, f\right)+\sum_{j=2}^{4} \delta\left(a_{j}, f\right)>3 .
$$

Further any two of $\left\{F\left(z, a_{j}\right)\right\}$ are not proportional. Then one of $\left\{a_{j}\right\}$ is a Picard exceptional value of $f$

THEOREM 3. Let $f(z)$ be the same as in the above theorem 2. Let $a_{1}, a_{2}, a_{3}, a_{4}$ and $a_{5}$ be five different finite numbers satisfying

$$
\begin{aligned}
& \Delta\left(a_{1}, f\right)+\delta\left(a_{2}, f\right)+\delta\left(a_{3}, f\right)+\delta\left(a_{4}, f\right)>3, \\
& \Delta\left(a_{1}, f\right)+\delta\left(a_{2}, f\right)+\delta\left(a_{3}, f\right)+\delta\left(a_{5}, f\right)>3 .
\end{aligned}
$$

Then at least two of $\left\{a_{j}\right\}$ are Picard exceptional values of $f$ or more precisely it occurs either

(a) $\delta\left(a_{1}, f\right)=\delta\left(a_{2}, f\right)=1$ and $\delta\left(a_{3}, f\right)=\delta\left(a_{4}, f\right)=\delta\left(a_{5}, f\right)>\frac{1}{2}$ or

(b) $\quad \delta\left(a_{1}, f\right)=\delta\left(a_{4}, f\right)=1$ and $\delta\left(a_{2}, f\right)=\delta\left(a_{3}, f\right)=\delta\left(a_{5}, f\right)>\frac{1}{2} \quad$ or

(c) $\delta\left(a_{2}, f\right)=\delta\left(a_{4}, f\right)=1$ and $\Delta\left(a_{1}, f\right)=\Delta\left(a_{3}, f\right)=\Delta\left(a_{5}, f\right)>\frac{1}{2}$ or

(d) $\delta\left(a_{2}, f\right)=\delta\left(a_{3}, f\right)=1$ and $\Delta\left(a_{1}, f\right)=\Delta\left(a_{4}, f\right)=\Delta\left(a_{5}, f\right)>\frac{1}{2}$ or

(e) $\quad \delta\left(a_{4}, f\right)=\delta\left(a_{5}, f\right)=1$ and $\Delta\left(a_{1}, f\right)=\Delta\left(a_{2}, f\right)=\Delta\left(a_{3}, f\right)>\frac{1}{2}$.

Further if there is another deficient value $a_{6}$ then

$$
\begin{aligned}
& \delta\left(a_{6}, f\right) \leqq 1-\delta\left(a_{3}, f\right) \quad \text { or } \\
& \delta\left(a_{6}, f\right) \leqq 1-\Delta\left(a_{1}, f\right)
\end{aligned}
$$

corresponding to the cases (a), (b) or (c), (d), (e). 
We need the following Lemma which is quite analogous to the expository Lemma in [1].

Here the author should like to thank sincerely Prof. M. Ozawa for his kind suggestions.

LEMMA. Let $g_{1}, g_{2}$ be two transcendental entire functions of finite order satisfying $\alpha g_{1}+\beta g_{2}=1, \alpha \beta \neq 0$. Then

$$
\Delta\left(0, g_{1}\right)+\delta\left(0, g_{2}\right) \leqq 1 .
$$

Proof. Suppose that $\Delta\left(0, g_{1}\right)+\delta\left(0, g_{2}\right)>1$. Therefore we have

$$
\delta\left(\infty, g_{2}\right)+\Delta\left(\frac{1}{\beta}, g_{2}\right)+\delta\left(0, g_{2}\right)>2,
$$

which contradicts the Nevanlinna defect relation

$$
\Delta\left(c_{1}, f\right)+\sum_{j=2}^{q} \delta\left(c_{j}, f\right) \leqq 2,
$$

where the $c_{\jmath}$ are any $q(\geqq 3)$ distinct complex numbers.

§ 2. Proof of Theorem 1. Niino-Ozawa's argument does work in our case. We firstly have

$$
F\left(z, a_{j}\right)=g_{\jmath}, \quad j=1,2,3
$$

and

$$
\sum_{j=1}^{4} \alpha_{j} g_{j}=1
$$

where

$$
\alpha_{1}=\frac{1}{\left(a_{1}-a_{2}\right)\left(a_{1}-a_{3}\right)}, \quad \alpha_{2}=-\frac{1}{\left(a_{1}-a_{2}\right)\left(a_{2}-a_{3}\right)}, \quad \alpha_{3}=\frac{1}{\left(a_{1}-a_{3}\right)\left(a_{2}-a_{3}\right)} .
$$

Now we suppose that all $g_{j}(z), j=1,2,3$, are transcendental. Differentiating both sides of (1) we have

$$
\sum_{j=1}^{3} \alpha_{j} \frac{g_{j}^{\prime}}{g_{\jmath}} g_{j}=0, \quad \sum_{j=1}^{3} \alpha_{j} \frac{g_{j}^{\prime \prime}}{g_{j}} g_{j}=0
$$

Assuming that $g_{1}, g_{2}, g_{3}$ are linearly independent, we have

$$
g_{1}=\frac{\Delta_{1}}{\alpha_{1} \Delta}, \quad g_{2}=\frac{\Delta_{2}}{\alpha_{2} \Delta},
$$

where 


$$
\begin{gathered}
\Delta=\left|\begin{array}{ccc}
1 & 1 & 1 \\
\frac{g_{1}^{\prime}}{g_{1}} & \frac{g_{2}^{\prime}}{g_{2}} & \frac{g_{3}^{\prime}}{g_{3}} \\
\frac{g_{1}^{\prime \prime}}{g_{1}} & \frac{g_{2}^{\prime \prime}}{g_{2}} & \frac{g_{3}^{\prime \prime}}{g_{3}}
\end{array}\right| \neq 0, \\
\Delta_{1}=\frac{g_{2}^{\prime}}{g_{2}} \frac{g_{3}^{\prime \prime}}{g_{3}}-\frac{g_{3}^{\prime}}{g_{3}} \frac{g_{2}^{\prime \prime}}{g_{2}}, \quad \Delta_{2}=\frac{g_{1}^{\prime \prime}}{g_{1}} \frac{g_{3}^{\prime}}{g_{3}}-\frac{g_{1}^{\prime}}{g_{1}} \frac{g_{3}^{\prime \prime}}{g_{3}} .
\end{gathered}
$$

Let

$$
2 \mu(r, A)=\frac{1}{2 \pi} \int_{0}^{2 \pi} \log A d \theta, \quad A=\max \left(1,\left|A_{1}\right|,\left|A_{2}\right|\right) .
$$

By Valiron's theorem [3]

$$
|T(r, f)-\mu(r, A)|=O(1) .
$$

Further we have

$$
2 \mu(r, A)=2 \mu(r, g)+O(1), \quad g=\max \left(1,\left|g_{1}\right|,\left|g_{2}\right|\right)
$$

Hence

$$
\begin{aligned}
\log g & =\log \max \left(1, \frac{\left|\Delta_{1}\right|}{\left|\alpha_{1} \Delta\right|}, \frac{\left|\Delta_{2}\right|}{\left|\alpha_{2} \Delta\right|}\right) \\
& \leqq \log ^{+}\left|\Delta_{1}\right|+\log ^{+}\left|\Delta_{2}\right|+\log ^{+} \frac{1}{|\Delta|}+O(1)
\end{aligned}
$$

Thus

$$
\begin{aligned}
2 \mu(r, g) & \leqq m\left(r, \Delta_{1}\right)+m\left(r, \Delta_{2}\right)+m\left(r, \frac{1}{\Delta}\right)+O(1) \\
& \leqq \sum_{j=1}^{3} N\left(r ; 0, g_{j}\right)+o\left(\sum_{j=1}^{3} m\left(r, g_{j}\right)\right)
\end{aligned}
$$

without exceptional set. Further for $j=1,2$

$$
m\left(r, g_{\jmath}\right) \leqq m(r, g)=2 \mu(r, g)
$$

and

$$
\begin{aligned}
m\left(r, g_{3}\right) & \leqq m\left(r, g_{1}\right)+m\left(r, g_{2}\right)+O(1) \\
& \leqq 4 \mu(r, g)+O(1) .
\end{aligned}
$$

Hence

$$
\sum_{j=1}^{3} m\left(r, g_{j}\right) \leqq 8 \mu(r, g)+O(1)
$$


Then we have

i.e.

$$
2 \mu(r, g) \leqq \sum_{j=1}^{3} N\left(r ; 0, g_{j}\right)+o(\mu(r, g))
$$

$$
1 \leqq \sum_{j=1}^{3} \frac{N\left(r ; 0, g_{j}\right)}{2 \mu(r, g)}+o(1)
$$

without exceptional set.

By the definition of Valiron deficiency

$$
\begin{aligned}
\lim _{r \rightarrow \infty} \frac{N\left(r ; 0, g_{1}\right)}{2 \mu(r, g)} & =\lim _{r \rightarrow \infty} \frac{N\left(r ; a_{1}, f\right)}{\mu(r, g)}=\lim _{r \rightarrow \infty} \frac{N\left(r ; a_{1}, f\right)}{T(r, f)} \\
& =1-\Delta\left(a_{1}, f\right),
\end{aligned}
$$

we have

$$
\begin{aligned}
1 & \leqq \lim _{r \rightarrow \infty} \frac{N\left(r ; 0, g_{1}\right)}{2 \mu(r, g)}+\sum_{j=2}^{3} \varlimsup_{r \rightarrow \infty} \frac{N\left(r ; 0, g_{j}\right)}{2 \mu(r, g)} \\
& =1-\Delta\left(a_{1}, f\right)+\sum_{j=2}^{3}\left(1-\delta\left(a_{j}, f\right)\right),
\end{aligned}
$$

which implies

$$
\Delta\left(a_{1}, f\right)+\sum_{\jmath=2}^{3} \delta\left(a_{\jmath}, f\right) \leqq 2
$$

This is absurd. Therefore $g_{1}, g_{2}, g_{3}$ are linearly dependent. Thus we have

$$
\beta_{1} g_{1}+\beta_{2} g_{2}+\beta_{3} g_{3}=0 \text {. }
$$

The above equation together with (1) gives

$$
\begin{array}{ll}
\gamma_{1} g_{1}+\gamma_{2} g_{2}=1, & \gamma_{1} \gamma_{2} \neq 0, \\
\gamma_{2} g_{2}+\gamma_{3} g_{3}=1, & \gamma_{2} \gamma_{3} \neq 0 .
\end{array}
$$

In the first place we consider the case (A). Now

$$
\begin{aligned}
m\left(r, g_{1}\right) & \leqq m(r, g)=2 \mu(r, g) \\
& \leqq \frac{1}{2 \pi} \int_{0}^{2 \pi} \log ^{+} \max \left(\left|\gamma_{1} g_{1}\right|, 1+\left|\gamma_{1} g_{1}\right|\right) d \theta+O(1) \\
& \leqq \frac{1}{2 \pi} \int_{0}^{2 \pi} \log ^{+}\left|g_{1}\right| d \theta+O(1) \\
& =m\left(r, g_{1}\right)+O(1) .
\end{aligned}
$$


Hence

$$
\left|m\left(r, g_{1}\right)-2 \mu(r, g)\right|=O(1)
$$

Further evidently

$$
\left|m\left(r, g_{1}\right)-m\left(r, g_{2}\right)\right|=O(1)
$$

Therefore

$$
\varliminf_{r \rightarrow \infty} \frac{N\left(r ; a_{1}, f\right)}{T(r, f)}=\lim _{r \rightarrow \infty} \frac{N\left(r ; 0, g_{1}\right)}{2 \mu(r, g)}=\lim _{r \rightarrow \infty} \frac{N\left(r ; 0, g_{1}\right)}{m\left(r, g_{1}\right)}
$$

This implies

$$
\Delta\left(a_{1}, f\right)=\Delta\left(0, g_{1}\right)
$$

Similarly we get

$$
\delta\left(a_{2}, f\right)=\delta\left(0, g_{2}\right)
$$

Hence we have

$$
\Delta\left(0, g_{1}\right)+\delta\left(0, g_{2}\right)=\Delta\left(a_{1}, f\right)+\delta\left(a_{2}, f\right)>1 .
$$

By virtue of Lemma we have a contradiction.

Secondly we consider the case (B). Similarly with the case (A), we have

$$
\delta\left(a_{j}, f\right)=\delta\left(0, g_{j}\right), \quad j=2,3 .
$$

Hence

$$
\delta\left(0, g_{2}\right)+\delta\left(0, g_{3}\right)=\delta\left(a_{2}, f\right)+\delta\left(a_{3}, f\right)>1,
$$

which gives similarly a contradiction.

These contradictions give that one of $\left\{g_{j}\right\}_{j=1}^{3}$ must be a polynomial: i.e. $(1 \mathrm{~A}) g_{1}$ is a polynomial, or (1B) $g_{2}$ is a polynomial.

Consider the case $(1 \mathrm{~A})$. Assume that $\alpha_{1} g_{1} \neq 1$.

From

$$
\alpha_{2} g_{2}+\alpha_{3} g_{3}=1-\alpha_{1} g_{1}
$$

we get

$$
\alpha_{2} \frac{g_{2}^{\prime}}{g_{2}} g_{2}+\alpha_{3} \frac{g_{3}^{\prime}}{g_{3}} g_{3}=-\alpha_{1} g_{1}^{\prime} .
$$

Thus, noticing that $g_{1}$ is a polynomial

$$
m\left(r, g_{2}\right) \leqq N\left(r ; 0, g_{2}\right)+N\left(r ; 0, g_{3}\right)+o(\mu(r, g)) .
$$

Then 


$$
1 \leqq \varlimsup_{r \rightarrow \infty} \sum_{j=2}^{3} \frac{N\left(r ; 0, g_{j}\right)}{m\left(r, g_{2}\right)} \leqq \sum_{j=2}^{3}\left(1-\delta\left(a_{j}, f\right)\right)
$$

by virtue of $\left|m\left(r, g_{2}\right)-2 \mu(r, g)\right|=O(\log r)$. Hence we have

$$
\delta\left(a_{2}, f\right)+\delta\left(a_{3}, f\right) \leqq 1,
$$

which is a contradiction. Hence $g_{2}$ and $g_{3}$ are linearly dependent. Consequently we have that $g_{2}$ and $g_{3}$ are polynomials respectively. Thus $A_{1}$ and $A_{0}$ are polynomials, which is absurd. This leads us to the following fact: $\alpha_{1} g_{1} \equiv 1$. Hence

$$
\delta\left(a_{2}, f\right)=\delta\left(a_{3}, f\right)>\frac{1}{2},
$$

and $\Delta\left(a_{1}, f\right)=\delta\left(a_{1}, f\right)=1$. This is the desired result (a). Next consider the case (1B). Assume that $\alpha_{2} g_{2} \neq 1$. Then we can obtain similarly to the case (1A)

$$
m\left(r, g_{1}\right) \leqq N\left(r ; 0, g_{1}\right)+N\left(r ; 0, g_{3}\right)+o(\mu(r, g))
$$

without exceptional set. Then we have

$$
\Delta\left(a_{1}, f\right)+\delta\left(a_{3}, f\right) \leqq 1,
$$

which is a contradiction. Consequently we get $\alpha_{2} g_{2} \equiv 1$. Hence we have

$$
\delta\left(a_{1}, f\right)=\delta\left(a_{3}, f\right) \quad \text { and } \quad \Delta\left(a_{1}, f\right)=\Delta\left(a_{3}, f\right)>\frac{1}{2},
$$

which is the desired result (b).

Assume that there is another deficiency $\delta\left(a_{4}, f\right)$ satisfying

$$
\Delta\left(a_{1}, f\right)+\delta\left(a_{2}, f\right)+\delta\left(a_{4}, f\right)>2 .
$$

Then we have

$$
\left(a_{2}-a_{4}\right) g_{1}+\left(a_{4}-a_{1}\right) g_{2}+\left(a_{1}-a_{2}\right) g_{4}=-\left(a_{2}-a_{4}\right)\left(a_{4}-a_{1}\right)\left(a_{1}-a_{2}\right) .
$$

By the above discussion we have in the case (a)

$$
\left(a_{2}-a_{3}\right) g_{1}=-\left(a_{2}-a_{3}\right)\left(a_{3}-a_{1}\right)\left(a_{1}-a_{2}\right),
$$

which shows

$$
\left(a_{4}-a_{1}\right) g_{2}+\left(a_{1}-a_{2}\right) g_{4}=-\left(a_{2}-a_{4}\right)\left(a_{4}-a_{3}\right)\left(a_{1}-a_{2}\right) \neq 0 .
$$

This implies a contradiction. Hence

$$
\begin{aligned}
\delta\left(a_{4}, f\right) & \leqq 2-\Delta\left(a_{1}, f\right)-\delta\left(a_{2}, f\right) \\
& =1-\delta\left(a_{2}, f\right) .
\end{aligned}
$$

In the case (b) we have 
DEFICIENCIES OF AN ENTIRE ALGEBROID FUNCTION OF FINITE ORDER

$$
\left(a_{3}-a_{1}\right) g_{2}=-\left(a_{3}-a_{1}\right)\left(a_{1}-a_{2}\right)\left(a_{2}-a_{3}\right),
$$

then

$$
\delta\left(a_{4}, f\right) \leqq 2-\Delta\left(a_{1}, f\right)-\delta\left(a_{2}, f\right)=1-\Delta\left(a_{1}, f\right) .
$$

§3. Proof of Theorem 2. We put

$$
g_{\jmath}=F\left(z, a_{j}\right), \quad j=1,2,3,4,
$$

and assume that all $g_{\jmath}, j=1,2,3,4$, are transcendental. Now we have

$$
\sum_{j=1}^{4} \alpha_{j} g_{\jmath}=1
$$

where

$$
\alpha_{\jmath}=1 / \prod_{\substack{k=1 \\ k \neq \jmath}}^{4}\left(a_{j}-a_{k}\right), \quad j=1,2,3,4 .
$$

Assume that $g_{1}, g_{2}, g_{3}$ and $g_{4}$ are linearly independent. Then the Wronskian does not vanish identically. By differentiating (3) we have

$$
\sum_{j=1}^{4} \alpha_{j} \frac{g_{j}^{(\mu)}}{g_{j}} g_{j}=0, \quad \mu=1,2,3
$$

We can solve (3) and (4). Then we get

$$
g_{\jmath}=\frac{\Delta_{j}}{\alpha_{j} \Delta}, \quad j=1,2,3,4
$$

where

$$
\Delta=\frac{1}{\prod_{j=1}^{4} g_{j}}\left|\begin{array}{cccc}
g_{1} & g_{2} & g_{3} & g_{4} \\
g_{1}^{\prime} & g_{2}^{\prime} & g_{3}^{\prime} & g_{4}^{\prime} \\
g_{1}^{\prime \prime} & g_{2}^{\prime \prime} & g_{3}^{\prime \prime} & g_{4}^{\prime \prime} \\
g_{1}^{\prime \prime \prime} & g_{2}^{\prime \prime \prime} & g_{3}^{\prime \prime \prime} & g_{4}^{\prime \prime \prime}
\end{array}\right|
$$

and $\Delta_{\jmath}$ is a polynomial of

$$
\frac{g_{1}^{(\mu)}}{g_{1}}, \cdots, \frac{g_{j-1}^{(\mu)}}{g_{j-1}}, \frac{g_{+1}^{(\mu)}}{g_{j+1}}, \cdots, \frac{g_{4}^{(\mu)}}{g_{4}}, \quad \mu=1,2,3 .
$$

Then 


$$
\begin{aligned}
\log g & =\log \max \left(1,\left|g_{1}\right|,\left|g_{2}\right|,\left|g_{3}\right|\right) \\
& \leqq \log ^{+} \frac{1}{|\Delta|}+\sum_{j=1}^{3} \log ^{+}\left|\Delta_{j}\right|+O(1) .
\end{aligned}
$$

Hence

$$
\begin{aligned}
3 \mu(r, g) & =\frac{1}{2 \pi} \int_{0}^{2 \pi} \log g d \theta=m(r, g) \\
& \leqq m\left(r, \frac{1}{\Delta}\right)+\sum_{j=1}^{3} m\left(r, \Delta_{j}\right)+O(1) \\
& \leqq \sum_{j=1}^{4} N\left(r ; 0, g_{j}\right)+o\left(\sum_{j=1}^{4} m\left(r, g_{j}\right)\right)
\end{aligned}
$$

without exceptional set. Further we have

$$
\sum_{j=1}^{4} m\left(r, g_{j}\right) \leqq 6 m(r, g)+O(1)
$$

and

$$
\mu(r, A)=\mu(r, g)+O(1)
$$

where

$$
A=\max \left(1,\left|A_{0}\right|,\left|A_{1}\right|,\left|A_{2}\right|\right) \text {. }
$$

Therefore

$$
\begin{aligned}
1 & \leqq \lim _{r \rightarrow \infty} \frac{N\left(r ; 0, g_{1}\right)}{3 \mu(r, g)}+\sum_{j=2}^{4} \varlimsup_{r \rightarrow \infty} \frac{N\left(r ; 0, g_{j}\right)}{3 \mu(r, g)} \\
& =1-\Delta\left(a_{1}, f\right)+\sum_{\jmath=2}^{4}\left(1-\delta\left(a_{\jmath}, f\right)\right)
\end{aligned}
$$

by virtue of Valiron's theorem. Thus

$$
\Delta\left(a_{1}, f\right)+\sum_{j=2}^{4} \delta\left(a_{j}, f\right) \leqq 3
$$

which is a contradiction. Hence we have the linear dependency of $g_{1}, g_{2}, g_{3}$ and $g_{4}$, that is,

$$
\sum_{j=1}^{4} \beta_{j} g_{\jmath}=0
$$

with constant $\left\{\beta_{j}\right\}$ not all zero. Here at least two of $\left\{\beta_{j}\right\}$ are not zero. Hence we may assume that 


$$
\text { (I) } \beta_{3} \beta_{4} \neq 0 \text { and } \beta_{4}=\alpha_{4} \text {, or (II) } \beta_{1} \beta_{2} \neq 0 \text { and } \beta_{1}=\alpha_{1} \text {. }
$$

We divide the cases (I), (II) into several subcases as follows:

\section{(I)}

Case 1) $\beta_{1} \beta_{2} \neq 0$.

(i) $\alpha_{1} \neq \beta_{1}, \quad \alpha_{2} \neq \beta_{2}, \quad \alpha_{3} \neq \beta_{3}$,

(ii) $\alpha_{1} \neq \beta_{1}, \quad \alpha_{2} \neq \beta_{2}, \quad \alpha_{3}=\beta_{3}$, $\alpha_{1} \beta_{2} \neq \alpha_{2} \beta_{1}$,

(iii) $\alpha_{1} \neq \beta_{1}, \quad \alpha_{2} \neq \beta_{2}, \quad \alpha_{3}=\beta_{3}$, $\alpha_{1} \beta_{2}=\alpha_{2} \beta_{1}$,

(iv) $\alpha_{1} \neq \beta_{1}, \quad \alpha_{2}=\beta_{2}, \quad \alpha_{3}=\beta_{3}$.

Case 2) $\beta_{1}=0, \quad \beta_{2} \neq 0$.

(i ) $\alpha_{2} \neq \beta_{2}, \quad \alpha_{3} \neq \beta_{3}$,

(ii) $\alpha_{2} \neq \beta_{2}, \quad \alpha_{3}=\beta_{3}$,

(iii) $\alpha_{2}=\beta_{2}, \alpha_{3}=\beta_{3}$.

Case 3) $\beta_{1}=\beta_{2}=0$.

(i) $\alpha_{3} \neq \beta_{3}$,

(ii) $\alpha_{3}=\beta_{3}$.

\section{(II)}

Case 4) $\beta_{3} \beta_{4} \neq 0$.

(i ) $\alpha_{2} \neq \beta_{2}, \quad \alpha_{3} \neq \beta_{3}, \quad \alpha_{4} \neq \beta_{4}$,

(ii) $\alpha_{2}=\beta_{2}, \quad \alpha_{3} \neq \beta_{3}, \quad \alpha_{4} \neq \beta_{4}$, $\alpha_{3} \beta_{4} \neq \alpha_{4} \beta_{3}$,

(iii) $\alpha_{2}=\beta_{2}, \quad \alpha_{3} \neq \beta_{3}, \quad \alpha_{4} \neq \beta_{4}$, $\alpha_{3} \beta_{4}=\alpha_{4} \beta_{3}$,

(iv) $\alpha_{2}=\beta_{2}, \quad \alpha_{3} \neq \beta_{3}, \quad \alpha_{4}=\beta_{4}$.

Case 5) $\quad \beta_{3}=0, \quad \beta_{4} \neq 0$.

(i) $\alpha_{2} \neq \beta_{2}, \quad \alpha_{4} \neq \beta_{4}$,

(ii) $\alpha_{2}=\beta_{2}, \quad \alpha_{4} \neq \beta_{4}$,

(iii) $\alpha_{2}=\beta_{2}, \quad \alpha_{4}=\beta_{4}$.

Case 6) $\beta_{3}=\beta_{4}=0$.

(i ) $\alpha_{2} \neq \beta_{2}$,

(ii) $\alpha_{2}=\beta_{2}$.

The cases 1), (iv); 2), (iii); 4), (iv) and 5), (iii) give trivially the desired result.

The cases 1), (i), (ii); 2), (i), (ii) and 3), (i) lead to an identity of the following type:

$$
\gamma_{1} g_{1}+\gamma_{2} g_{2}+\gamma_{3} g_{3}=1, \quad \gamma_{1} \gamma_{2} \gamma_{3} \neq 0 .
$$

The cases 4), (i), (ii); 5), (i), (ii) and 6), (i) also lead to an identity of the following type:
$(\mathrm{A})^{\prime}$
$\gamma_{2} g_{2}+\gamma_{3} g_{3}+\gamma_{4} g_{4}=1, \quad \gamma_{2} \gamma_{3} \gamma_{4} \neq 0$.

The cases 1), (iii) and 4), (iii) lead to

$$
\gamma_{1} g_{1}+\gamma_{2} g_{2}=1, \quad \gamma_{3} g_{3}+\gamma_{4} g_{4}=1, \quad \gamma_{1} \gamma_{2} \gamma_{3} \gamma_{4} \neq 0 .
$$

The cases 3), (ii) and 6), (ii) lead to

$$
\alpha_{1} g_{1}+\alpha_{2} g_{2}=1, \quad \alpha_{3} g_{3}+\alpha_{4} g_{4}=0
$$


and

$(\mathrm{C})^{\prime}$

$$
\alpha_{1} g_{1}+\alpha_{2} g_{2}=0, \quad \alpha_{3} g_{3}+\alpha_{4} g_{4}=1
$$

respectively.

By our assumption the cases $(C)$ and $(C)^{\prime}$ may be omitted.

In the first place we suppose that (A) occurs. Assuming the linear independency of $g_{1}, g_{2}, g_{3}$, we can apply the same method as in the above and then we arrive at a contradiction. Hence $g_{1}, g_{2}, g_{3}$ are linearly dependent. This and (A) imply

$$
\begin{aligned}
& \delta_{1} g_{1}+\delta_{2} g_{2}=1, \quad \delta_{1} \delta_{2} \neq 0, \quad \text { or } \\
& \delta_{2} g_{2}+\delta_{3} g_{3}=1, \quad \delta_{2} \delta_{3} \neq 0 .
\end{aligned}
$$

Considering the cases (a) or (b), we arrive at a contradiction in either case by the Lemma. Hence we can say that one of $\left\{g_{j}\right\}_{j=1,2,3}$ is a polynomial.

Similarly consider the case $(\mathrm{A})^{\prime}$, we can obtain that $g_{2}, g_{3}, g_{4}$ are linearly dependent. This and $(\mathrm{A})^{\prime}$ imply for example

$$
\delta_{3} g_{3}+\delta_{4} g_{4}=1, \quad \delta_{3} \delta_{4} \neq 0 .
$$

In this case we have the same desired result.

Secondly we suppose that (B) occurs. Let $G=\max \left(1,\left|g_{1}\right|,\left|g_{3}\right|\right)$. Then

$$
m(r, g) \leqq m(r, G)+O(1) \leqq m(r, g)+O(1) .
$$

Further

$$
m\left(r, g_{j}\right) \leqq m(r, G)+O(1), \quad j=2,4
$$

Hence

$$
\begin{aligned}
m(r, G) & \leqq m\left(r, g_{1}\right)+m\left(r, g_{3}\right) \\
& \leqq \sum_{j=1}^{4} N\left(r ; 0, g_{j}\right)+o(m(r, G))
\end{aligned}
$$

without exceptional set. This leads to the following contradictory inequality

$$
\Delta\left(a_{1}, f\right)+\sum_{\jmath=2}^{4} \delta\left(a_{\jmath}, f\right) \leqq 3 .
$$

Thus either $g_{1}, g_{2}$ or $g_{3}, g_{4}$ are proportional, which is absurd.

§4. Proof of Theorem 3. We set also

$$
g_{\jmath}=F\left(z, a_{j}\right), \quad j=1, \cdots, 5,
$$

and 


$$
\beta_{1} g_{1}+\beta_{2} g_{2}+\beta_{3} g_{3}+\beta_{5} g_{5}=1
$$

and suppose that all $g_{j}(z), j=1, \cdots, 5$, are transcendental. Therefore the reasoning in the proof of Theorem 2 leads to the following cases:

$$
\text { (i) (C) and (5), (ii) (C)' and (5). }
$$

Since the case (ii) can be handled quite similarly, we only consider the case (i). Since $\alpha_{1} \beta_{2} \neq \beta_{1} \alpha_{2}$, we have

$$
\left(\alpha_{1} \beta_{2}-\alpha_{2} \beta_{1}\right) g_{2}+\alpha_{1} \beta_{3} g_{3}+\alpha_{1} \beta_{5} g_{5}=\alpha_{1}-\beta_{1} \neq 0
$$

or

$$
\left(\alpha_{2} \beta_{1}-\alpha_{1} \beta_{2}\right) g_{1}+\alpha_{2} \beta_{3} g_{3}+\alpha_{2} \beta_{5} g_{5}=\alpha_{2}-\beta_{2} \neq 0 .
$$

Thus we obtain a desired contradiction in either case. Hence one of $\left\{g_{j}\right\}$ is a polynomial.

Consequently we have the following fact: At least one of $\left\{g_{j}\right\}_{j=1}^{5}$ must be a polynomial, that is

(3A) $g_{1}$ is a polynomial, or $(3 \mathrm{~B}) g_{2}$ is a polynomial, or (3C) $g_{4}$ is a polynomial.

Firstly we consider the case (3A). Further assume that the other $g_{\jmath}$ are transcendental. If $\alpha_{1} g_{1} \neq 1$, then the identity (3) implies

$$
\alpha_{2} g_{2}+\alpha_{3} g_{3}+\alpha_{4} g_{4}=1-\alpha_{1} g_{1} .
$$

By the same method as in the proof of Theorem 1, this case gives a contradiction. Thus we have the existence of a polynomial among $g_{2}, g_{3}, g_{4}$. In this case we get

$$
\begin{aligned}
& \delta\left(a_{1}, f\right)=\delta\left(a_{2}, f\right)=1 \quad \text { for example or } \\
& \delta\left(a_{1}, f\right)=\delta\left(a_{4}, f\right)=1 .
\end{aligned}
$$

The case (a) leads to

$$
\begin{aligned}
& \alpha_{3} g_{3}+\alpha_{4} g_{4}=1-\alpha_{1} g_{1}-\alpha_{2} g_{2}, \\
& \beta_{3} g_{3}+\beta_{5} g_{5}=1-\beta_{1} g_{1}-\beta_{2} g_{2} .
\end{aligned}
$$

By virtue of the argument in the case (1A) of Theorem 1, we have the linear dependency of $g_{3}$ and $g_{4}, g_{3}$ and $g_{5}$ respectively, that is,

$$
\delta\left(a_{3}, f\right)=\delta\left(a_{4}, f\right)=\delta\left(a_{5}, f\right)>\frac{1}{2} .
$$

The case (b) leads to

$$
\begin{aligned}
& \alpha_{2} g_{2}+\alpha_{3} g_{3}=1-\alpha_{1} g_{1}-\alpha_{4} g_{4}=0 \\
& \beta_{2} g_{2}+\beta_{3} g_{3}+\beta_{5} g_{5}=1-\beta_{1} g_{1},
\end{aligned}
$$


which yeilds also

$$
\delta\left(a_{2}, f\right)=\delta\left(a_{3}, f\right)=\delta\left(a_{5}, f\right)>\frac{1}{2}
$$

by virtue of our standard method. If $\alpha_{1} g_{1} \equiv 1$, then we have

$$
\beta_{2} g_{2}+\beta_{3} g_{3}+\beta_{5} g_{5}=1-\frac{\beta_{1}}{\alpha_{1}}
$$

by (5), where

$$
\beta_{1}=\frac{1}{\left(a_{1}-a_{2}\right)\left(a_{1}-a_{3}\right)\left(a_{1}-a_{5}\right)} .
$$

Hence

$$
1-\frac{\beta_{1}}{\alpha_{1}}=\frac{a_{4}-a_{5}}{a_{1}-a_{5}} \neq 0 .
$$

Therefore we can prove the existence of another polynomial among $g_{2}, g_{3}, g_{5}$ Also we have

$$
\begin{array}{ll}
(\mathrm{a})^{\prime} & \delta\left(a_{1}, f\right)=\delta\left(a_{2}, f\right)=1 \quad \text { for example or } \\
(\mathrm{b})^{\prime} & \delta\left(a_{1}, f\right)=\delta\left(a_{5}, f\right)=1 .
\end{array}
$$

The case (a)' leads to

$$
\begin{aligned}
& \alpha_{3} g_{3}+\alpha_{4} g_{4}=-\alpha_{2} g_{2}, \\
& \beta_{3} g_{3}+\beta_{5} g_{5}=1-\frac{\beta_{1}}{\alpha_{1}}-\beta_{2} g_{2}=0,
\end{aligned}
$$

which is absurd.

The case (b)' also leads to

$$
\begin{aligned}
& \alpha_{2} g_{2}+\alpha_{3} g_{3}+\alpha_{4} g_{4}=0, \\
& \beta_{2} g_{2}+\beta_{3} g_{3}=1-\frac{\beta_{1}}{\alpha_{1}}-\beta_{5} g_{5}=0
\end{aligned}
$$

In this case we obtain a part of the desired result:

$$
\delta\left(a_{2}, f\right)=\delta\left(a_{3}, f\right)=\delta\left(a_{4}, f\right)>\frac{1}{2} .
$$

Secondly we consider the case (3B). Further assume that the other $g_{j}$ are transcendental. If $\alpha_{2} g_{2} \neq 1$, then we have the existence of a polynomial among $g_{1}, g_{3}, g_{4}$ from the identity

$$
\alpha_{1} g_{1}+\alpha_{3} g_{3}+\alpha_{4} g_{4}=1-\alpha_{2} g_{2}
$$


by the standard method. Thus we get

$$
\begin{array}{ll}
\delta\left(a_{2}, f\right)=\delta\left(a_{4}, f\right)=1 & \text { or } \\
\delta\left(a_{2}, f\right)=\delta\left(a_{3}, f\right)=1 & \text { or } \quad \text { (a). }
\end{array}
$$

The case (c) leads to

$$
\begin{aligned}
& \alpha_{1} g_{1}+\alpha_{3} g_{3}=1-\alpha_{2} g_{2}-\alpha_{4} g_{4}=0, \\
& \beta_{1} g_{1}+\beta_{3} g_{3}+\beta_{5} g_{5}=1-\beta_{2} g_{2},
\end{aligned}
$$

which yeilds

$$
\begin{aligned}
& \delta\left(a_{1}, f\right)=\delta\left(a_{3}, f\right)=\delta\left(a_{5}, f\right) \text { or } \\
& \Delta\left(a_{1}, f\right)=\Delta\left(a_{3}, f\right)=\Delta\left(a_{5}, f\right)>\frac{1}{2} .
\end{aligned}
$$

The case $(\mathrm{d})$ leads to

$$
\begin{aligned}
& \alpha_{1} g_{1}+\alpha_{4} g_{4}=1-\alpha_{2} g_{2}-\alpha_{3} g_{3}=0 \\
& \beta_{1} g_{1}+\beta_{5} g_{5}=1-\beta_{2} g_{2}-\beta_{3} g_{3}=0
\end{aligned}
$$

which provides

$$
\begin{aligned}
& \delta\left(a_{1}, f\right)=\delta\left(a_{4}, f\right)=\delta\left(a_{5}, f\right) \quad \text { or } \\
& \Delta\left(a_{1}, f\right)=\Delta\left(a_{4}, f\right)=\Delta\left(a_{5}, f\right)>\frac{1}{2} .
\end{aligned}
$$

If $\alpha_{2} g_{2} \equiv 1$, then we have only the following case, that is,

$(\mathrm{c})^{\prime}$

$$
\delta\left(a_{2}, f\right)=\delta\left(a_{5}, f\right)=1
$$

by virtue of the argument in the above case: $\alpha_{1} g_{1} \equiv 1$. In this case we get

$$
\begin{aligned}
& \delta\left(a_{1}, f\right)=\delta\left(a_{3}, f\right)=\delta\left(a_{4}, f\right) \quad \text { or } \\
& \Delta\left(a_{1}, f\right)=\Delta\left(a_{3}, f\right)=\Delta\left(a_{4}, f\right)>\frac{1}{2} .
\end{aligned}
$$

Finally we consider the case (3C). If $\alpha_{4} g_{4} \neq 1$, then the reasoning in the above cases leads to the following cases:

$$
\text { (b) or (c). }
$$

If $\alpha_{4} g_{4} \equiv 1$, we have

$$
\begin{aligned}
& \alpha_{1} g_{1}+\alpha_{2} g_{2}+\alpha_{3} g_{3}=0, \\
& \beta_{1} g_{1}+\beta_{2} g_{2}+\beta_{3} g_{3}+\beta_{5} g_{5}=1 .
\end{aligned}
$$


Hence we get

$$
\begin{array}{lll}
\gamma_{1} g_{1}+\gamma_{3} g_{3}+\gamma_{5} g_{5}=1, & \gamma_{1} \gamma_{3} \gamma_{5} \neq 0 & \text { or } \\
\gamma_{2} g_{2}+\gamma_{3} g_{3}+\gamma_{5} g_{5}=1, & \gamma_{2} \gamma_{3} \gamma_{5} \neq 0 . &
\end{array}
$$

Thus by the standard method we have the existence of a polynomial among $g_{1}, g_{3}, g_{5}$ or $g_{2}, g_{3}, g_{5}$ respectively. These cases give

(b) or $(\mathrm{c})^{\prime}$

$$
\begin{aligned}
& \delta\left(a_{3}, f\right)=\delta\left(a_{4}, f\right)=1 \quad \text { or } \\
& \delta\left(a_{4}, f\right)=\delta\left(a_{5}, f\right)=1 .
\end{aligned}
$$

For example the case (e) leads to

which yields

$$
\begin{aligned}
& \gamma_{1} g_{1}+\gamma_{3} g_{3}=1-\gamma_{5} g_{5}=0, \\
& \alpha_{1} g_{1}+\alpha_{2} g_{2}+\alpha_{3} g_{3}=0,
\end{aligned}
$$

$$
\begin{aligned}
& \delta\left(a_{1}, f\right)=\delta\left(a_{2}, f\right)=\delta\left(a_{3}, f\right) \quad \text { or } \\
& \Delta\left(a_{1}, f\right)=\Delta\left(a_{2}, f\right)=\Delta\left(a_{3}, f\right)>\frac{1}{2} .
\end{aligned}
$$

Thus the proof of Theorem 3 is complete.

§5. Applying the method in the proof of Theorem 3, we have the following

THEOREM 4. Let $f(z)$ be the same as in the theorem 2. Let $a_{1}, a_{2}, a_{3}, a_{4}$ and $a_{5}$ be five different finite numbers satisfying

$$
\begin{aligned}
& \delta\left(a_{1}, f\right)+\delta\left(a_{2}, f\right)+\delta\left(a_{3}, f\right)+\Delta\left(a_{4}, f\right)>3, \\
& \delta\left(a_{1}, f\right)+\delta\left(a_{2}, f\right)+\delta\left(a_{3}, f\right)+\Delta\left(a_{5}, f\right)>3 .
\end{aligned}
$$

Then at least two of $a_{j}$ are Picard exceptional values of $f$ or more precisely it occurs either

$$
\begin{aligned}
& \delta\left(a_{1}, f\right)=\delta\left(a_{2}, f\right)=1 \quad \text { for example and } \\
& \Delta\left(a_{3}, f\right)=\Delta\left(a_{4}, f\right)=\Delta\left(a_{5}, f\right)>\frac{1}{2} \quad \text { or }
\end{aligned}
$$

$$
\begin{aligned}
& \delta\left(a_{1}, f\right)=\delta\left(a_{4}, f\right)=1 \quad \text { for example and } \\
& \delta\left(a_{2}, f\right)=\delta\left(a_{3}, f\right)=\delta\left(a_{5}, f\right)>\frac{1}{2} \quad \text { or } \\
& \delta\left(a_{4}, f\right)=\delta\left(a_{5}, f\right)=1 \quad \text { and } \\
& \delta\left(a_{1}, f\right)=\delta\left(a_{2}, f\right)=\delta\left(a_{3}, f\right)>\frac{1}{2} .
\end{aligned}
$$




\section{REFERENCES}

[1] Nino, K., And Ozawa, M., Deficiencies of an entire algebroid function. Kōdai Math. Sem. Rep. 22 (1970), 98-113.

[2] Nino, K., And Ozawa, M., Deficiencies of an entire algebroid function, II. Kōdai Math. Sem. Rep. 22 (1970), 178-187.

[3] Valiron, G., Sur la dérivée des fonctions algébroïdes. Bull. Soc. Math. 59 (1931), 17-39.

Department of Mathematics,

Chiba University. 